CERN-PPE/92-191

5 November 1992

\title{
Diffractive production of charmed strange mesons by neutrinos and antineutrinos
}

Big Bubble Chamber Neutrino Collaboration

A.E. Asratyan ${ }^{8}$, M. Aderholz ${ }^{9}$, V.V. Ammosov ${ }^{7}$, W. Burkot ${ }^{5}$, E.F. Clayton ${ }^{6}$,

T. Coghen ${ }^{5}$, O. Erriquez ${ }^{1}$, G.S. Gapienko ${ }^{7}$, V.A. Gapienko ${ }^{7}$, J. Guy $^{10}$,

D. Hantke ${ }^{9}$, G.T. Jones ${ }^{2}$, V.S. Kaftanov ${ }^{8}$, U.F. Katz ${ }^{9}$, J. Kern ${ }^{9}$,

V.A. Korotkov ${ }^{7}$, S.P. Krutchinin ${ }^{8}$, M.A. Kubantsev ${ }^{8}$, P. Marage ${ }^{3}$, D.B. Miller ${ }^{6}$, M.M. Mobayyen ${ }^{6}$, D.R.O. Morrison ${ }^{4}$, M. Neveu ${ }^{11}$, N. Schmitz $^{9}$, K. Varvell ${ }^{2 \dagger}$,

W. Venus ${ }^{10}$, W. Wittek ${ }^{9}$, V.G. Zaetz ${ }^{7}$

Abstract. The diffractive production of charmed strange $D_{s}^{*}$ and possibly $D_{s}$ mesons by neutrinos and antineutrinos on nucleons in hydrogen, deuterium and neon targets is observed. The slope parameter of the $t$ distribution is $3.3 \pm 0.8(\mathrm{GeV})^{-2}$. The production rate per charged current neutrino interaction with an isoscalar target times the $D_{s}^{+} \rightarrow \phi \pi^{+}$branching fraction is $(1.03 \pm 0.27) \times 10^{-4}$.

[1] Dipartimento di Fisica dell'Universita e Sezione INFN, I-70126 Bari, Italy.

[2] University of Birmingham, Birmingham B15 2TT, UK.

[3] Inter-University Institute for High Energies, ULB-VUB, B-1050 Brussels, Belgium.

[4] CERN, CH-1211 Geneva 23, Switzerland.

[5] Institute of Nuclear Physics, PL-30055 Cracow, Poland.

[6] Imperial College of Science and Technology, London, SW7 2AZ, UK.

[7] Institute of High Energy Physics, Serpukhov, Russia

[8] Institute of Theoretical and Experimental Physics, Moscow, Russia.

[9] Max-Planck-Institut für Physik, D-8000 München 40, Germany.

[10] Rutherford Appleton Laboratory, Chilton, Didcot, OX11 0QX, UK.

[11] DPhPE, Centre d'Etudes Nucléaires, Saclay, F-91191 Gif sur Yvette, France.

† Now at ANSTO, Menai NSW 2234, Australia.

Submitted to Physics Letters B 


\section{Introduction}

In a neutrino induced reaction, the $c \bar{s}$ pair from the Cabibbo-favoured transition $W^{+} \rightarrow c \bar{s}$ may rescatter as a whole off a target nucleon (or nucleus), emerging in the final state as an on-mass-shell charmed strange meson[1]-[3]. Experimentally, little is known as the diffractive contribution is only a small fraction of all charm production. One $D_{s}(1969)$ event (interpreted as diffractive in the present analysis) was found in hydrogen[4] and one $D_{s}^{*-}(2111)$ in neon[5] data from BEBC. This paper describes a search for the $D_{s}^{*}$ and $D_{s}$ diffractive production in a large sample of charged current interactions including the data from four bubble chamber experiments using wide band neutrino and antineutrino beams.

In the BEBC experiments WA21 (hydrogen fill), WA25 (deuterium), and WA59 (heavy neon-hydrogen mix), the data were collected using a very similar wide band, horn focused beam, with mean neutrino (antineutrino)-induced event energy near 50(40) GeV. The experiment E180 used the 15-ft bubble chamber filled with a heavy $\mathrm{Ne}-\mathrm{H}_{2}$ mix and exposed to a wide band antineutrino beam under conditions essentially similar to WA59. The individual data samples are discussed in more detail in references [6]-[9]. The combined statistics of the four experiments are some 57000 neutrino and 52000 antineutrino charged current events with a muon with momentum above $4 \mathrm{GeV}$ detected by the external muon identifier. The hydrogen and deuterium data are called the 'light fill' data below.

\section{Selection of the $D_{s}$ and $D_{s}^{*}$ candidates}

With the $D_{s}^{*}$ decays being $100 \%$ radiative $\left(D_{s}^{*} \rightarrow \gamma D_{s}\right)$, the selection of the $D_{s}$ weak decay candidates is crucial for both the $D_{s}$ and the $D_{s}^{*}$ searches. As the $D_{s}(1969)$ is too short lived to be directly observed in these large bubble chambers, one can rely only on the mass selection in a number of decay channels. The weak $D_{s}$ decays selected are :

$$
\begin{aligned}
& D_{s}^{+} \rightarrow K^{+} \overline{K^{0}} \\
& D_{s}^{+} \rightarrow \phi \pi^{+}, \phi \rightarrow K^{+} K^{-} \\
& D_{s}^{+} \rightarrow \overline{K^{* 0}} K^{+}, \overline{K^{* 0}} \rightarrow K^{-} \pi^{+} \\
& D_{s}^{+} \rightarrow K^{*+} \overline{K^{0}}, K^{*+} \rightarrow K^{0} \pi^{+} \\
& D_{s}^{+} \rightarrow \overline{K^{* 0}} K^{*+}, \overline{K^{* 0}} \rightarrow K^{-} \pi^{+}, K^{*+} \rightarrow K^{0} \pi^{+}
\end{aligned}
$$

(plus the conjugate states for the $D_{s}^{-}$production by antineutrinos). These decays include most experimentally studied $D_{s}$ decays which have two kaons and no neutral pion in the final state[10]. The observed $\phi 3 \pi$ decay is not included since the combinatorial background in this channel is too high and, 
anyway, the branching fraction is only about $40 \%$ of the $\phi \pi$ fraction. These arguments equally apply to the non-resonant $K^{+} K^{-} \pi^{+}$contribution which is also ignored. The $\phi \pi^{+}, K^{+} \overline{K^{0}}, \overline{K^{* 0}} K^{+}$, and $K^{*+} \overline{K^{0}}$ branching fractions of the $D_{s}^{+}$ are known to be nearly the same within some $20 \%$, while the $\overline{K^{* 0}} K^{*+}$ decays are nearly twice as frequent. The reference $\phi \pi^{+}$branching fraction itself is probably around $3-4 \%[10],[11]$. The decays with neutral pions such as $D_{s}^{+} \rightarrow \phi \rho^{+}$, $\rho^{+} \rightarrow \pi^{+} \pi^{0}$ which are not efficiently registered in the light fill are not selected for the sake of uniform acceptance throughout the combined data.

To restrict the $D_{s}$ mass resolution, we require the fractional momentum uncertainty for charged particles and $K_{s}^{0}$ (reconstructed from their decay to $\pi^{+} \pi^{-}$) to be below 0.25 and 0.07 for the neon and the light fill data, respectively. Charged kaons are not identified in the bubble chamber; instead the kaon hypothesis is tried for every hadron not unambiguously identified as a pion or a proton. The $K^{*}$ candidates are taken within $60 \mathrm{MeV}$ of the nominal $K^{*}$ mass. The $\phi$ candidates are selected as possible $K^{+} K^{-}$pairs with mass within 3 standard deviations of $1019.4 \mathrm{MeV}$. The mass uncertainty includes both the propagated charged $K$ momentum and angle errors and the $\phi$ decay width (the two contributions are of the same order of magnitude).

To reduce the combinatorial background to the (quasi-)two-particle decays (1-5), the forward-backward decay topologies are removed by retaining only events with $|\cos \theta|<0.9$ where $\theta$ is the angle between the $D_{s}$ Lorentz boost direction from the laboratory system and the neutral decay particle (resonance) momentum, as measured in the $D_{s}$ rest frame. Since the $D_{s}$ is spinless, the acceptance of this selection is 0.9 , while the background is approximately halved. Without further selections, decay channel (3) would remain a major source of combinatorial background as the $\overline{K^{* 0}}$ selection is far less restrictive than the $\phi$ selection in channel (2). In this $\mathrm{P}$-wave decay the $\cos \beta$ distribution, where $\beta$ is the angle between the $K^{+}$and $K^{-}$momenta in the $\overline{K^{* 0}}$ rest frame, is proportional to $(\cos \beta)^{2}$. Accordingly, only events with $|\cos \beta|>0.6$ having an acceptance of 0.78 are retained for channel (3).

For each $D_{s}$ candidate (denoted as $X$ ), we compute the mass $m_{X}$ from the measured 3-momenta of the decay products, the mass measurement uncertainty $\sigma$, and the variable $\delta$ - the distance between $m_{X}$ and $m_{D_{s}}$ in terms of the uncertainty: $\delta=\left(m_{X}-1969 \mathrm{MeV}\right) / \sigma$. Here $\sigma$ is computed by propagating all relevant measurement errors; the typical values are some 10 and $40 \mathrm{MeV}$ for the light and heavy fills, respectively.

For a $D_{s}$ candidate $X$ and a gamma to be taken as forming $D_{s}^{*}$ candidate, the mass difference $m_{\gamma X}-m_{X}$ is required to lie within 3 standard deviations of $141.5 \pm 1.9 \mathrm{MeV}[10]$. The mass difference uncertainty is again computed by propagating the momentum and angle measurement errors of all the particles; the typical values are some 5 to $10 \mathrm{MeV}$ for the light fill and $25 \mathrm{MeV}$ for the neon. 


\section{Selection of diffractive candidates}

The final state of the diffractive interaction is formed by a muon, a charmed strange meson $\left(D_{s}^{*}\right.$ or $\left.D_{s}\right)$, and a recoil nucleon (proton or neutron). So we require candidates to have an identified muon and perhaps an identified proton with all other observed charged particles, $K^{0}$ decays and gamma conversions included in the $D_{s}$ or the $D_{s}^{*}$ decay chain. In neon, additional protons from intranuclear reinteractions or nuclear breakup are allowed. Occasional reinteractions of neutrals, commonly called n-stars, are ignored for all data.

The absolute value of the 4-momentum transfer squared to the nucleon, $t$, and its minimal value for the given event kinematics, $t_{m i n}$, are estimated by constraining the event kinematics to $D_{s}$ (or $D_{s}^{*}$ ) production off a target nucleon at rest ( $D_{s}^{*}$ if an extra gamma is seen, $D_{s}$ if not), ignoring the recoil nucleon (if seen). If the $D_{s}^{*}$ decay gamma is not detected (which is usually the case with the light fill), the $t$ value estimated in this way is distorted with respect to the true value. The sign and magnitude of the distortion depend on the (missed) gamma production angle in the $D_{s}^{*}$ rest frame. The mean difference between the two $t$ values is around $0.1(\mathrm{GeV})^{2}$.

The advantage of the hydrogen data (WA21) is the possibility of a tight kinematic constraint under certain conditions: if all final particles are observed and correctly identified, the differences $\left(E-P_{l}\right)$ between the particles' energies and longitudinal momenta should sum up to the target mass (equal to proton mass $M_{p}$ ). If the masses of the final particles are overestimated (in our case, if some charged pions are assigned as kaons), the calculated target mass can exceed $M_{p}$, signalling that the mass assignment is incorrect. The effectiveness of this kinematic constraint in case of the $D_{s}^{*}$ production is hampered by the loss of most $D_{s}^{*}$ decay gammas in hydrogen. For each WA21 event containing a $D_{s}$ or $D_{s}^{*}$ candidate, we compute the visible value of the target mass, $M_{t}$, taking the mass assignments from the $D_{s}$ decay hypothesis. We also compute the corresponding uncertainty, $\sigma_{M_{t}}$, by propagating the measurement uncertainties. The candidate decays with computed target mass $M_{t}$ significantly above the proton mass $M_{p}$, i.e. $M_{t}-M_{p}$ larger than $3 \sigma_{M_{t}}$, are ignored. A lower limit on the visible target mass is not imposed in order not to exclude possible $D_{s}^{*}$ candidates.

\section{The diffractive signal}

Unlike the deep-inelastic cross sections, the diffractive cross sections are predicted to be the same for incident neutrinos and antineutrinos and for proton and neutron targets. Figure 1 shows the momentum transfer, $t$, plotted against the normalised variable, $\delta$. A cluster of events is seen near the $D_{s}(1969)$ mass, $\delta=0$, at $t$ values below some $1(\mathrm{GeV})^{2}$. Figure 2 shows the mass distributions of the selected combinations $X$ with $t$ below 2.0 and $1.2(\mathrm{GeV})^{2}$ (cross hatched). 
The narrow peak at the $D_{s}$ mass is formed by the light-fill candidates having the smallest mass errors. The mass intervals used on figure 2 are such that the maximum of the $D_{s}$ contribution is expected between 1.96 and $1.985 \mathrm{GeV}$. The dark distribution is for the candidates with $t$ below $1.2(\mathrm{GeV})^{2}$ from the central $D_{s}$ mass region with $|\delta|$ below 3 . As individual mass errors vary by a factor of 20 , the $\delta$ rather than the $m_{X}$ distribution is analysed to estimate the magnitude of the effect.

Figures $3 \mathrm{a}$ and $3 \mathrm{~b}$ show the antineutrino and neutrino contributions to the $\delta$ distribution for $t$ below 2.0 and $1.2(\mathrm{GeV})^{2}$ (cross hatched) plotted separately. Figure 3c shows both combined. A fit of the combined $\delta$ plot with $t$ below $1.2(\mathrm{GeV})^{2}$ to a Gaussian plus a linear function yields $\delta=-0.1 \pm 0.4$ for the peak position, $1.5 \pm 0.4 \delta$ units for the rms deviation, and $22.9 \pm 5.6$ events for the magnitude of the effect. Within errors, no effect is seen at higher $t$ values: for $t$ below $2(\mathrm{GeV})^{2}, 21.7 \pm 5.6$ events above background are observed. A similar fit of the separate antineutrino (Figure $3 \mathrm{a}$ ) and neutrino (Figure $3 \mathrm{~b}$ ) contributions with $t$ below $1.2(\mathrm{GeV})^{2}$ gives $12.4 \pm 4.4$ and $9.8 \pm 3.6$ events above background, respectively.

For the comparison of production rates, the hydrogen contributions to the $\bar{\nu}$ and $\nu$ charged current samples are renormalised to an isoscalar target $(I)$ in accordance with reference [12]. The equivalent $\bar{\nu} I$ and $\nu I$ statistics then amount to some 47900 and 66700 charged current events, respectively. The ratio of the observed neutrino and antineutrino rates per charged current interaction on an isoscalar target $\left[(1.5 \pm 0.5) \times 10^{-4}\right.$ and $(2.6 \pm 0.9) \times 10^{-4}$, respectively] is compatible with $1: 2$, as implied by the equality of diffractive cross sections and the ratio of inclusive cross sections.

To probe the energy dependence, all the charged current events in each neutrino energy interval are rescaled to the equivalent number of $\nu I$ events. The energy loss due to the unobserved neutral hadrons is corrected for by a statistical method based on transverse momentum balance[12], [13]. In the neutrino energy intervals 10 to 30,30 to 50 , and 50 to $200 \mathrm{GeV}$ the observed rate per charged current $\nu I$ interaction is $(1.8 \pm 0.7) \times 10^{-4},(1.3 \pm 0.6) \times 10^{-4}$, and $(1.6 \pm 0.7) \times 10^{-4}$, respectively, so that no variation is detected at the available statistical level. Theoretically, no steep variation of this relative rate in the 10 to $200 \mathrm{GeV}$ neutrino energy interval is expected[3].

The separate contributions to Figure $3 \mathrm{c}$ of the $D_{s}$ decay channels (1-5) are shown in Figure 4. Within errors, the observed contributions to the signal are in a proportion compatible with the $D_{s}$ branching fractions and registration efficiencies (see Section 8). 


\section{Possible contributions from non-strange $D$ decays}

Theoretically, diffractive production of charmed non-strange $D^{+}$and $D^{*+}$ mesons is Cabibbo-suppressed as compared to $D_{s}^{*+}$ and $D_{s}^{+}$. Nevertheless the possibility that some of the signal seen here is due to diffractive $D^{+}$and $D^{*+}$ production rather than $D_{s}^{+}$and $D_{s}^{*+}$ cannot be ignored. To test this our first approach was to

(i) select combinations with $t$ below $1.2(\mathrm{GeV})^{2}$ contributing to Figure 3c,

(ii) for each final system $(1,2,3,5)$ above, choose the mass assignment $X_{+}$compatible with the Cabibbo-favoured $D^{+}$meson decay with one kaon in the final state (as there are no charged kaons in (4), these final states are not considered here),

(iii) compute the mass of the combination, $m_{+}=m_{X_{+}}$, the mass measurement error, $\sigma_{+}$, and $\delta_{+}=\left(m_{+}-m_{D^{+}}\right) / \sigma_{+}$.

Figure 5a shows the $\delta_{+}$distribution with the distribution for the peak events with $|\delta|$ below 3 dark. Since much of the effect in Figure 3 is reflected into an enhancement near $\delta_{+}=0$ observed in Figure 5a, no definite conclusion about the $D^{+}$contribution can be drawn.

An alternative approach is to study the $D^{*+}$ production which, by the argument of spin counting, is expected to be dominant:

$$
D^{*+} \rightarrow D^{0} \pi^{+}, D^{0} \rightarrow K^{-} \pi^{+} \text {or } \overline{K^{0}} \pi^{+} \pi^{-}
$$

These decays are selected in the events with no extra particles apart from a muon and identified protons. Again, for each $D^{0}$ decay candidate $X_{0}$ we compute the mass, $m_{0}$, mass measurement error, $\sigma_{0}$, and $\delta_{0}=\left(m_{0}-m_{D^{0}}\right) / \sigma_{0}$. The observed mass difference $\left(m_{X_{0} \pi^{+}}-m_{0}\right)$ is required to lie within 3 deviations of $m_{D^{*+}}-m_{D^{0}}$ (the uncertainty is again computed by error propagation). Figure $5 \mathrm{~b}$ shows the $\delta_{0}$ distribution (with no selection on $t$ ). No candidates are seen within 3 deviations of the $D^{0}$ mass. With the measured branching fractions of the $D^{*+}[14]$ and $D^{0}[10]$ mesons, we estimate that the fraction of all produced charged $D^{*}$ mesons which can be registered in the bubble chamber through the decay chain 6 is about $4 \%$, similar to the fraction of $D_{s}$ mesons registered (see Section 8 ). This analysis suggests that the non-strange charmed meson contribution to the selected diffractive effect is negligibly small.

\section{Effects of proton misidentification}

Protons are distinguished in the bubble chamber by range or energy loss consistent with their measured curvature, and also by their consistent ionisation 
for the light fills. Protons with ranges above about $1 \mathrm{~cm}$ and momenta below about $750 \mathrm{MeV}$ are distinguished efficiently[15]. Losses of very slow protons (below $150 \mathrm{MeV}$ momentum for light fill and $350 \mathrm{MeV}$ for neon) do not affect our analysis as these are treated as unseen nucleons. The proton identification efficiency falls for proton momenta over about $750 \mathrm{MeV}$. Possible consequences include the reduction of the observed production rate and the softening of the observed $t$ distribution.

In order to recover events with unidentified protons, we select events with one positive particle not identified as a proton (neon target events with more identified protons attributed to intranuclear reinteractions or nuclear breakup are also accepted). In neon, the track length of this positive particle is required not to exceed the stopping proton range. In the light fill, we consider all the positive particles (not already identified as protons) with momentum over $750 \mathrm{MeV}$ as possible protons. We treat this 'possible proton positive' (PPP) sample in exactly the same way as the main sample (see Section 4 ). When computing the visible target mass, $M_{t}$, the extra positive particle is assigned a proton mass.

The distributions of $\delta$ with the PPP events included are shown in Figure $3 \mathrm{~d}$. For the complete $\delta$ distributions of Figure 3d, the fitted magnitude of the effect above background is $21.0 \pm 6.0$ events for $t$ below $2.0(\mathrm{GeV})^{2}$ (or $23.3 \pm 5.8$ events for $t$ below $\left.1.2(\mathrm{GeV})^{2}\right)$. Comparison with the corresponding numbers for the main sample alone $(21.7 \pm 5.6$ and $22.9 \pm 5.6$, see Section 4$)$ shows that, within errors, no leakage of the signal from the main sample to the PPP sample is detected at the existing statistical level.

\section{$7 \quad$ The constrained and $D_{s}^{*}$ candidates and $t$ dis- tribution}

The following two (possibly overlapping) categories of candidate events are of special interest:

(i) the hydrogen events with visible target mass compatible with that of a proton, $\left|M_{t}-M_{p}\right|<3 \sigma_{M_{t}}$;

(ii) events with the $D_{s}^{*}$ decay gamma seen in the bubble chamber (see Section 2).

The entries for this limited subsample are ringed on figure 1, including the additional PPP events. Six events with $m_{X}$ within 3 deviations of $m_{D_{s}}$ are observed (none of them PPP). These events are detailed in Table 1. Several kinematic variables of the $D_{s}$ candidate are shown. For the $D_{s}^{*}$ candidates, the measured $D_{s}^{*}-D_{s}$ mass difference is also given. When the recoil proton is detected, $t_{p}$ is an alternative evaluation of momentum transfer squared using the measured proton momentum. For the hydrogen data, the visible target mass $M_{t}$ 
is compared with $M_{t^{\prime}}$ computed with all charged mesons taken as pions. Events 2 and 4 in Table 1 were first selected in references [5] and [4], respectively.

Events $1-3$ in Table 1 are the $D_{s}^{*}$ candidates with decay gamma measured in neon where the registration efficiency is the highest. Events 4-6 are the constrained hydrogen $D_{s}$ candidates, with no extra gamma required for energymomentum conservation. If these are instead examples of $D_{s}^{*}$ production with an unobserved decay gamma, its contribution to $E-P_{l}$ could be between $0-69$, $0-5$, and $0-30 \mathrm{MeV}$ for the events 4-6, respectively (depending on the decay angle). Although an unobserved gamma can never be excluded with absolute certainty, these events suggest that the pseudoscalar charmed strange mesons are diffractively produced along with the vector ones. Apart from the reduced background level (there are no additional events in the $D_{s}$ mass region, $|\delta|$ below 3 , at higher $t$ values), another advantage of this limited subsample is that the observed $t$ value is much less affected by the $D_{s}^{*}-D_{s}$ ambiguity. Therefore we use this limited subsample, rather than all the $D_{s}$ candidates shown in Figure 1, for the estimate of the momentum-transfer-squared distribution slope parameter.

Assuming that $t^{\prime}=t-t_{\min }$ behaves like $e^{-B t^{\prime}}$ and that the $t^{\prime}$ slopes are the same for the $D_{s}^{*}$ and $D_{s}$ production, an average over the 6 ringed events in the cluster on Figure 1 gives the $t^{\prime}$ distribution slope parameter $B=(3.3 \pm 0.8)(\mathrm{GeV})^{-2}$.

\section{Production rate}

Since the diffractive cross section is unchanged by the $\mathrm{n}-\mathrm{p}$ or neutrino - antineutrino exchanges, we choose to normalise the signal to the total equivalent number of charged current neutrino interactions with an isoscalar target $I$ (see Section 4). The equivalent statistics of the four experiments are 165300 charged current $\nu I$ interactions.

The $D_{s}$ branching fraction ratios[10] used for the cross section estimate are shown in Table 2. The detector registration efficiency for each $D_{s}$ decay channel includes the relevant $\phi$ and $K^{*}$ branching fractions and the $K_{s}^{0}$ registration efficiency in the bubble chamber taken as $0.52[9],[16]$. From the numbers in table 2 , the quantity (acceptance $\times$ branching ratio) $/ B R\left(D_{s} \rightarrow \phi \pi^{+}\right.$) is estimated as $0.24 \pm 0.04,0.45,0.45 \pm 0.05,0.05 \pm 0.01$, and $0.19 \pm 0.05$ for the $D_{s}$ decay channels (1-5), respectively. The signal obtained by fitting the overall (including PPP) $\delta$ distribution with $t$ below $1.2(\mathrm{GeV})^{2}$ is used for the production rate estimate since, within errors, no effect is observed at higher $t$ values. Hence the $\left(D_{s}+D_{s}^{*}\right)$ diffractive production rate, $R$, per charged current $\nu I$ interaction times the $D_{s}^{+} \rightarrow \phi \pi^{+}$branching fraction is $R \times B R\left(D_{s} \rightarrow \phi \pi^{+}\right)=(1.03 \pm 0.27) \times 10^{-4}$. For $B R\left(D_{s} \rightarrow \phi \pi^{+}\right)=0.037 \pm 0.012$ as estimated in reference [11], this becomes $R=(2.8 \pm 1.1) \times 10^{-3}$.

In reference $[5]$ the inclusive $D_{s}^{*-}$ production rate per charged current $\bar{\nu} I$ interaction times the $D_{s} \rightarrow \phi \pi$ branching fraction had been estimated as 
$(2.3 \pm 0.7) \times 10^{-3}$. Assuming that the inclusive $D_{s}^{*}$ production cross sections in the $\nu I$ and $\bar{\nu} I$ interactions are equal (as predicted by the model of vector meson dominance[1]) and taking into account the factor 2 between the total charged current $\nu I$ and $\bar{\nu} I$ cross sections, we conclude that about $10 \%$ of all charmed strange mesons are produced diffractively in neutrino interactions.

\section{Conclusion}

Several sets of neutrino data from bubble chambers have been successfully merged to provide over 109000 charged current events with complete measurements of all secondary charged hadrons, allowing for the detection of rare processes which are statistically beyond reach of the individual experiments.

In this paper, the diffractive production of charmed strange $D_{s}^{*}$ and $D_{s}$ mesons by neutrinos and antineutrinos off a target nucleon is studied. The diffractive candidates are selected with a muon, $D_{s}^{*}$ or $D_{s}$, and possible recoil nucleon. Most of the $D_{s}$ decays to final states with two kaons, but without a $\pi^{0}$ or neutrino, are selected.

The production of vector $\left(D_{s}^{*}\right)$ and possibly pseudoscalar $\left(D_{s}\right)$ mesons is observed. The slope parameter of the momentum-transfer-squared distribution is $(3.3 \pm 0.8)(\mathrm{GeV})^{-2}$. The observed $D_{s}^{*}+D_{s}$ production rate per charged current neutrino interaction with an isoscalar target times the $D_{s}^{+} \rightarrow \phi \pi^{+}$ branching fraction shows no visible energy dependence in our energy range (10$200 \mathrm{GeV})$ and its average value is $(1.03 \pm 0.27) \times 10^{-4}$.

Acknowledgements. We would like to thank the E180, WA21, WA25 and WA59 collaborations for allowing us to include their data, and G.Van Apeldoorn and our other colleagues in the wide band beam experiments who helped make this collaboration of collaborations possible. 


\section{References}

[1] B.A. Arbuzov et al., Sov. J. Nucl. Phys. 21, 682 (1975); Phys. Lett. 58B, 64 (1975).

[2] M.K. Gaillard et al., Nucl. Phys. B102, 326 (1976).

[3] M.-S. Chen et al., Nucl. Phys. B118, 345 (1977).

[4] J. Kern, Diploma thesis, MPI Munich, September 1986 (unpublished).

[5] A.E. Asratyan et al., Phys. Lett. 257B, 525 (1991).

[6] G.T. Jones et al., Z. Phys. C51, 11 (1991).

[7] D. Allasia et al., Z. Phys. C37, 527 (1988).

[8] K. Varvell et al., Z. Phys. C36, 1 (1987).

[9] V.V. Ammosov et al., Nucl. Phys. B177, 365 (1981).

[10] Particle Data Group, Phys. Rev. D45, Number 11 (1992).

[11] M. Daoudi et al., Phys. Rev. D45, 3965 (1992).

[12] M. Aderholz et al., Phys. Lett. 173B, 211 (1986).

[13] J. Guy et al., Z. Phys. C36, 337 (1987).

[14] B. Gittelman, CLNS 91/1112 (1991).

[15] E. Matsinos et al., Z. Phys. C44, 79 (1989); J. Guy et al., Phys. Lett. 229B, 421 (1989); D. Allasia et al., Nucl. Phys. B343, 285 (1990).

[16] S. Willocq et al., Z. Phys. C53, 207 (1992); G.T.Jones et al, 'Neutral strange particle production in neutrino and antineutrino charged current interactions on protons' MPI Munich preprint MPI-PhE/92-11, submitted to Z. Phys. C. 


\section{Figure captions}

Figure 1: $t$ vs $\delta=\left(m_{X}-1969 \mathrm{MeV}\right) / \sigma$ for the main-sample $D_{s}$ and $D_{s}^{*}$ candidates selected as in Sections 2 and 3 (crosses). Candidates also passing the selections in Section 7 are ringed, (including additional PPP candidates found by the selections in Section 6, filled circles).

Figure 2: Distribution of the mass $m_{X}$ for the $D_{s}$ and $D_{s}^{*}$ candidates with $t<2.0$ and $<1.2(\mathrm{GeV})^{2}$ (cross hatched) and for the central $D_{s}$ mass region, $|\delta|<3$, with $t<1.2(\mathrm{GeV})^{2}$ (dark).

Figure 3: The $\delta=\left(m_{X}-1969 \mathrm{MeV}\right) / \sigma$ distributions of the $D_{s}$ and $D_{s}^{*}$ candidates with $t<2$ and $<1.2(\mathrm{GeV})^{2}$ (cross hatched): (a) antineutrinos; (b) neutrinos; (c) antineutrinos and neutrinos; (d) antineutrinos and neutrinos, PPP events added.

Figure 4: The separate contributions to figure $3(\mathrm{c})$ with $t<1.2(\mathrm{GeV})^{2}$ of the $D_{s}$ decay channels (1-5).

Figure 5: (a) The $\delta_{+}=\left(m_{+}-m_{D^{+}}\right) / \sigma_{+}$distribution of the $X_{+}$combinations obtained from the $X$ combinations contributing to the $\delta$ distribution of Figure 3c $\left(t<1.2(\mathrm{GeV})^{2}\right)$ by choosing the charged meson mass assignments consistent with the Cabibbo-favoured decays of the non-strange charged $D$ mesons. The dark $X_{+}$combinations correspond to $X$ in the central $D_{s}$ mass region, $|\delta|<3$; (b) the $\delta_{0}=\left(m_{0}-m_{D^{0}}\right) / \sigma_{0}$ distribution of the $X_{0}$ combinations compatible with $D^{*+} \rightarrow D^{0} \pi^{+}, D^{0} \rightarrow X_{0}$ decays (see Section 5). 
Table 1 The $D_{s}^{*}$ and $D_{s}$ candidates with $|\delta|<3$ satisfying the Section 7 selections. $E_{\nu}$ is the neutrino energy, $Q^{2}$ the 4-momentum transfer squared to the hadrons, $m_{X}$ the mass, $\delta=\left(m_{X}-1969 \mathrm{MeV}\right) / \sigma, p_{X}$ the total momentum of $X$, $t$ the 4 -momentum transfer squared to the nucleon ignoring the recoil nucleon while $t_{p}$ uses the measured proton and $t^{\prime}$ is with all charged mesons taken as pions; $M_{t}$ is the visible target mass (see text).

\begin{tabular}{|c|c|c|c|c|c|c|c|}
\hline $\begin{array}{c}E_{\nu} \\
\mathrm{GeV}\end{array}$ & $\begin{array}{c}Q^{2} \\
(\mathrm{GeV})^{2}\end{array}$ & $\begin{array}{c}m_{X} \\
\mathrm{MeV}\end{array}$ & $\delta$ & $\begin{array}{c}p_{X} \\
\mathrm{GeV}\end{array}$ & $\begin{array}{c}t \\
(\mathrm{GeV})^{2}\end{array}$ & $\begin{array}{c}t_{p} \\
(\mathrm{GeV})^{2}\end{array}$ & $\begin{array}{c}t^{\prime} \\
(\mathrm{GeV})^{2}\end{array}$ \\
\hline \multicolumn{8}{|c|}{1 WA59 event $8734472 D_{s}^{*+} \rightarrow \gamma D_{s}^{+}, D_{s}^{+} \rightarrow \overline{K^{* 0}} K^{+}$} \\
\hline 157.0 & 5.27 & $1970 \pm 46$ & 0.0 & 143.6 & 0.33 & 0.22 & 0.33 \\
\hline \multicolumn{8}{|c|}{$m_{\gamma X}-m_{X}=130 \pm 23 \mathrm{MeV}$} \\
\hline \multicolumn{8}{|c|}{2 WA59 event $8654942 D_{s}^{*-} \rightarrow \gamma D_{s}^{-}, D_{s}^{-} \rightarrow K^{*-} K^{0}$} \\
\hline 21.9 & 2.03 & $1991 \pm 30$ & 0.7 & 15.5 & 0.61 & 0.14 & 0.57 \\
\hline \multicolumn{8}{|c|}{$m_{\gamma X}-m_{X}=135 \pm 22 \mathrm{MeV}$} \\
\hline \multicolumn{8}{|c|}{$3 \quad$ E180 event $1359737 D_{s}^{*-} \rightarrow \gamma D_{s}^{-}, D_{s}^{-} \rightarrow \phi \pi^{-}$} \\
\hline 91.7 & 1.41 & $1927 \pm 31$ & -1.4 & 7.6 & 0.33 & 0.0 & 0.14 \\
\hline \multicolumn{8}{|c|}{$m_{\gamma X}-m_{X}=254 \pm 48 \mathrm{MeV}$} \\
\hline \multicolumn{8}{|c|}{4 WA21 event $7993546 D_{s}^{-} \rightarrow \phi \pi^{-}$} \\
\hline 118.0 & 3.51 & $1981 \pm 21$ & 0.5 & 7.3 & 0.51 & 0.51 & 0.03 \\
\hline \multicolumn{8}{|c|}{$M_{t}=938.0 \pm 10.8 \mathrm{MeV}, M_{t^{\prime}}=861.6 \pm 11.0 \mathrm{MeV}$} \\
\hline \multicolumn{8}{|c|}{5 WA21 event $2823083 D_{s}^{+} \rightarrow \overline{K^{* 0}} K^{+}$} \\
\hline 109.3 & 2.34 & $1956 \pm 9$ & -1.6 & 73.5 & 0.67 & 0.48 & 0.47 \\
\hline \multicolumn{8}{|c|}{$M_{t}=941.2 \pm 3.4 \mathrm{MeV}, M_{t^{\prime}}=930.8 \pm 3.4 \mathrm{MeV}$} \\
\hline \multicolumn{8}{|c|}{6 WA21 event $2834838 D_{s}^{+} \rightarrow \overline{K^{0}} K^{+}$} \\
\hline 28.9 & 1.71 & $1935 \pm 15$ & -2.3 & 12.1 & 0.34 & 0.34 & 0.28 \\
\hline \multicolumn{8}{|c|}{$M_{t}=938.0 \pm 3.2 \mathrm{MeV}, M_{t^{\prime}}=898.6 \pm 3.2 \mathrm{MeV}$} \\
\hline
\end{tabular}

Table 2 The relative branching fractions, registration efficiencies and angular cut acceptances for the selected $D_{s}$ decay channels.

\begin{tabular}{|c|c|c|c|}
\hline $\begin{array}{c}D_{s} \\
\text { decay } \\
\text { mode }\end{array}$ & $\frac{B R(\text { decay })}{B R\left(\phi \pi^{+}\right)}$ & $\begin{array}{c}\text { detector } \\
\text { registration } \\
\text { efficiency }\end{array}$ & $\begin{array}{c}\text { acceptance } \\
\text { of angular } \\
\text { cuts }\end{array}$ \\
\hline$K^{+} \overline{K^{0}}$ & $1.01 \pm 0.16$ & 0.26 & 0.90 \\
$\phi \pi^{+}$ & 1 & 0.50 & 0.90 \\
$\overline{K^{* 0}} K^{+}$ & $0.95 \pm 0.10$ & 0.67 & 0.71 \\
$\frac{K^{*+}}{K^{0}}$ & $1.20 \pm 0.25$ & 0.05 & 0.90 \\
$\overline{K^{* 0}} K^{*+}$ & $1.80 \pm 0.50$ & 0.12 & 0.90 \\
\hline
\end{tabular}




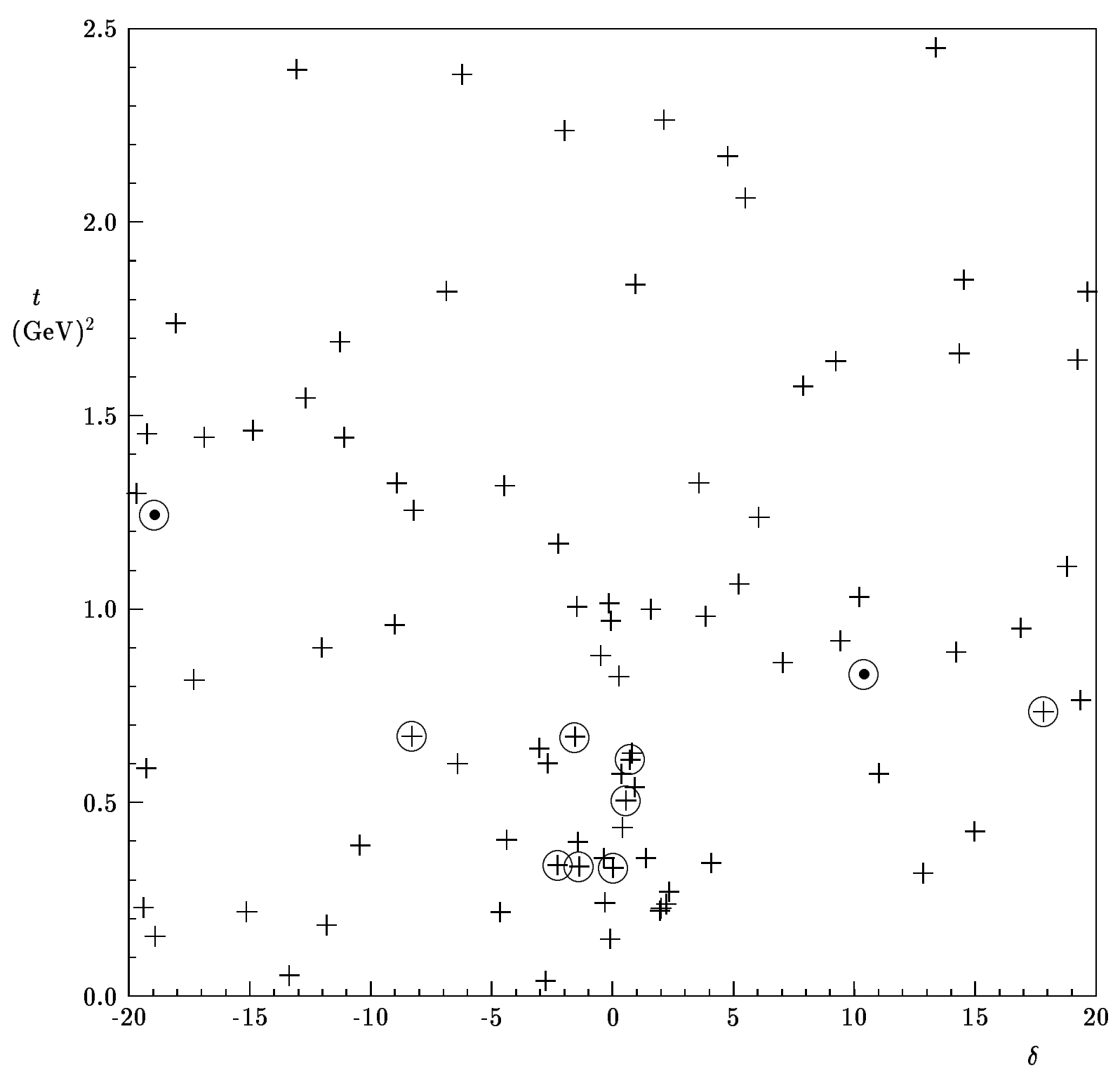

Figure 1 


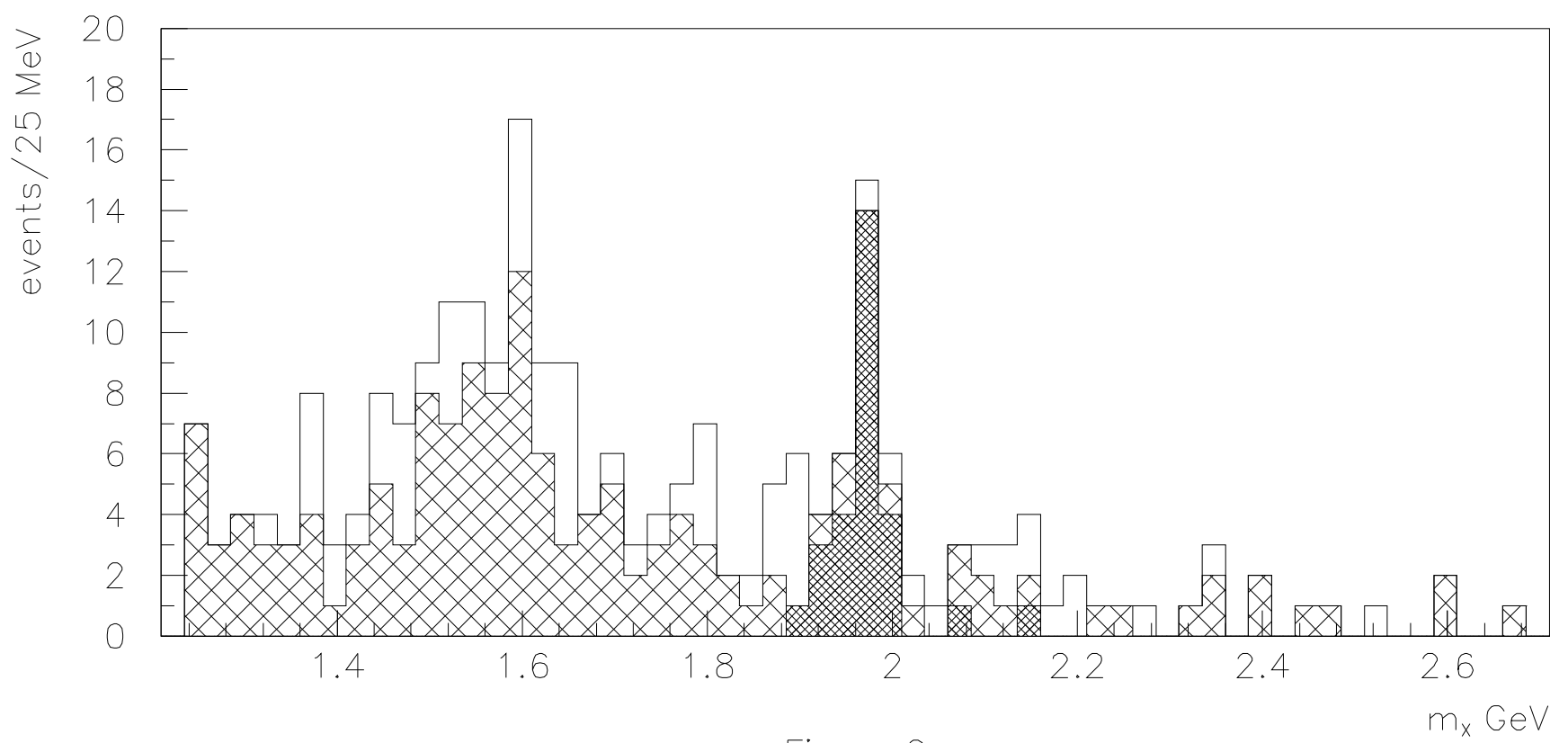

Figure 2 

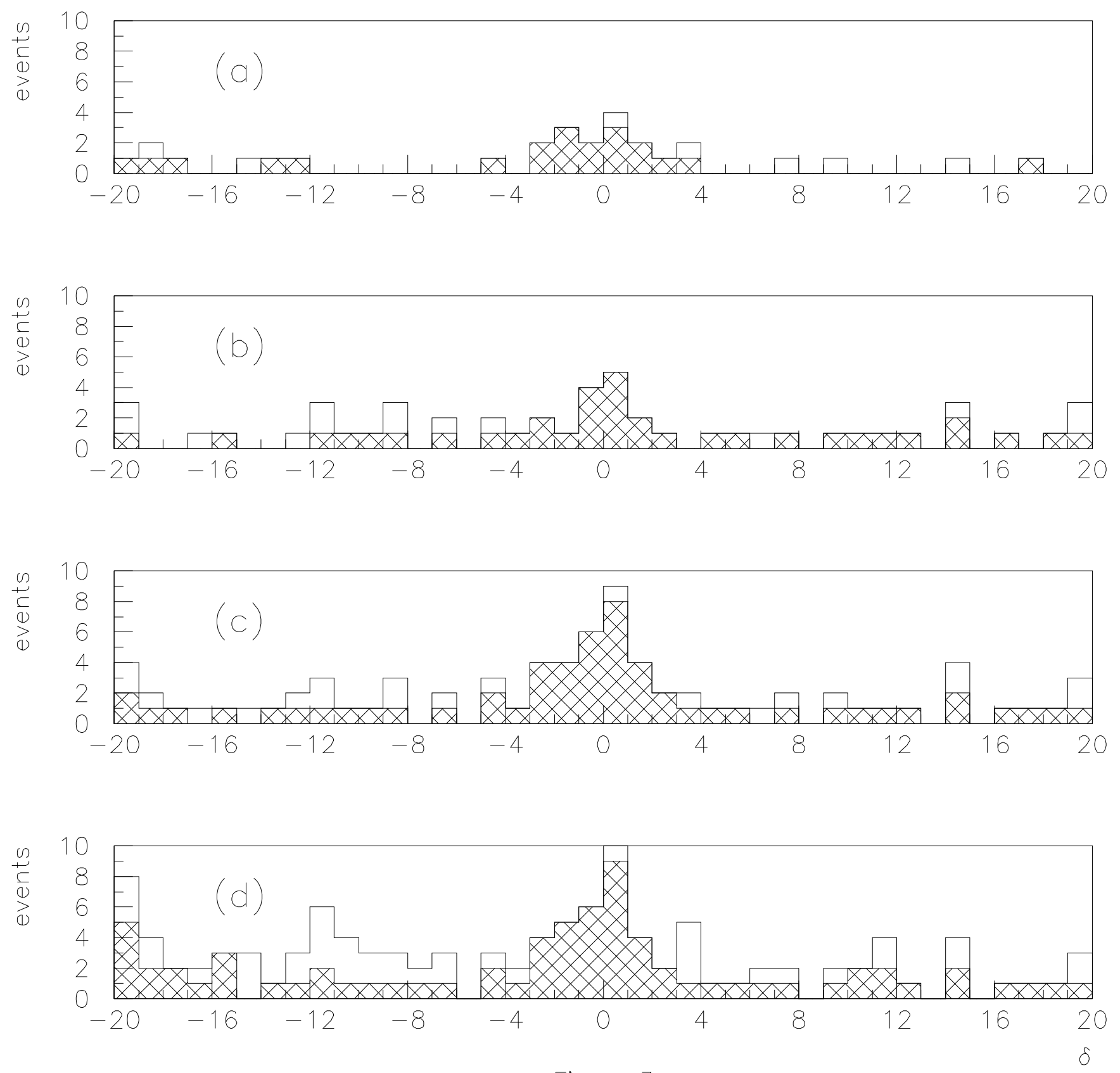

Figure 3 

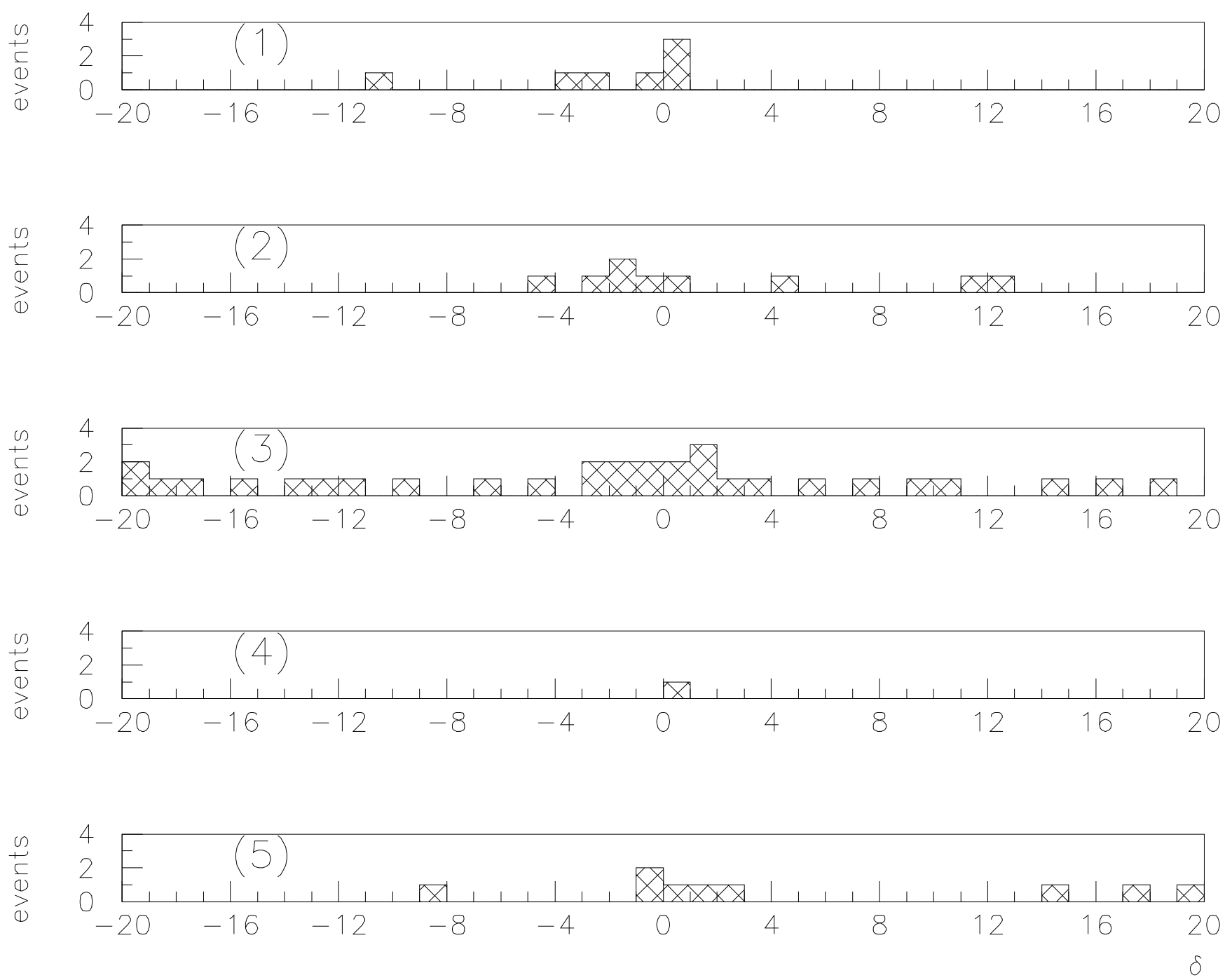

Figure 4 

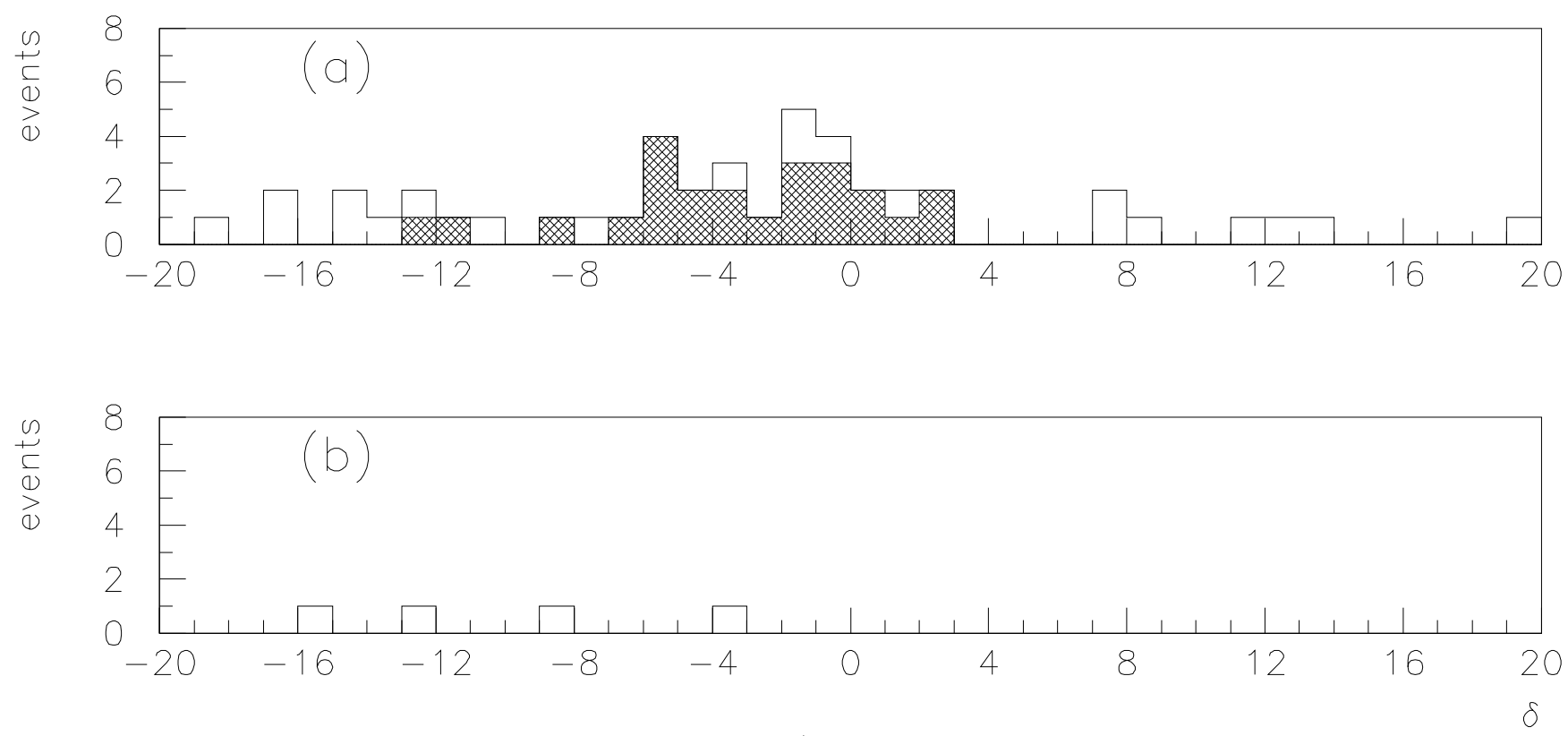

Figure 5 